\title{
Amplitude Contrast Imaging: High Resolution Electron Microscopy Using a Spherical and Chromatic Aberration Corrected TEM
}

\author{
J.G. Wen, D.J. Miller, R.E. Cook, N.J. Zaluzec \\ Electron Microscopy Center, Argonne National Laboratory, IL 60439 USA
}

High-resolution transmission electron microscopy (HREM) is a powerful technique to study materials at atomic resolution. For a conventional TEM, the Scherzer defocus is typically used to maximize phase contrast and resolution. For a TEM with a spherical aberration $\left(\mathrm{C}_{\mathrm{s}}\right)$ image corrector, Jia et al introduced the negative $\mathrm{C}_{\mathrm{s}}$ imaging (NCSI) method to balance the contrast and resolution [1]. This paper shows when $\mathrm{C}_{\mathrm{s}}$ is corrected close to zero, chromatic aberration $\left(\mathrm{C}_{\mathrm{c}}\right)$ correction improves resolution without compromising contrast and enables amplitude-contrast imaging (ACI) in HREM.

Employing aberration correctors commercial instruments can now routinely decrease the magnitude of $\mathrm{C}_{\mathrm{s}}$ towards zero and dramatically improve resolution. Unfortunately this also dramatically reduces the magnitude of phase contrast transfer function (PCTF) as shown in Fig. 1A. To balance resolution and phase contrast, Jia et al introduced the NCSI method in which a negative $\mathrm{C}_{\mathrm{s}}$ value and a corresponding Lichte defocus are chosen to gain strong phase contrast with sufficient resolution and facilitate direct structural mapping [1]. However when $\mathrm{C}_{c}$ is uncorrected, the $\mathrm{C}_{\mathrm{c}}$ damping envelope still limits the ultimate resolution. In an uncorrected $\mathrm{C}_{\mathrm{c}}$ configuration, $\mathrm{C}_{\mathrm{s}}$ corrected and uncorrected HREM images are still dominated by phase contrast as long as $\mathrm{C}_{\mathrm{c}}$ is of significant magnitude. This is shown in Fig. $2 \mathrm{D}$ and $2 \mathrm{E}$, which presents simulated HREM images of a $\mathrm{CaTiO}_{3} / \mathrm{BaTiO}_{3}$ interface using $\mathrm{C}_{\mathrm{s}}=-40 \mathrm{~m}=-40$ ace $\mathrm{u}$ both with $\mathrm{C}_{\mathrm{c}}=1.5 \mathrm{~mm}$ and show close correlation to the phase image (Fig. 2B) of the exit wave.

With $\mathrm{C}_{\mathrm{s}}$ close to zero, we have found that $\mathrm{C}_{\mathrm{c}}$ correction can be used to play an important role to improve resolution for both phase-contrast and amplitude-contrast HREM as a small $\mathrm{C}_{c}$ value can improve resolution without compromising phase contrast. In addition, $\mathrm{C}_{\mathrm{c}}$ correction has an even more substantial effect on the amplitude contrast transfer function (ACTF) where $\mathrm{C}_{c}$ correction offers the possibility to exploit ACI in HREM. The green filled curve in Fig. $1 \mathrm{C}$ shows ACTF with small values of $\mathrm{C}_{\mathrm{s}}$ and $\mathrm{C}_{\mathrm{c}}$ $\left(\mathrm{C}_{\mathrm{c}}=0.1 \mathrm{~mm}\right)$. For these conditions, the magnitude of ACTF is close to 1 over a wide range of spatial frequency nearly up to the information limit of the microscope. Under such ACI conditions (i.e. both $\mathrm{C}_{\mathrm{s}}$ and $\mathrm{C}_{\mathrm{c}}$ are small), the experimental image has a strong correlation with the calculated amplitude image (Fig. 2C) of the exit wave phase. An example of the correlation is illustrated in Fig. 2F, which shows the simulated image using our ACI condition.

Under ACI conditions, atomic resolution channeling contrast (ARCC) also can be realized [2]. Fig. 3 presents an amplitude contrast HREM image of a $\left(\mathrm{BaTiO}_{3}\right)_{4} /\left(\mathrm{CaTiO}_{3}\right)_{4}$ ferroelectric superlatice. In this image, ARCC between $\mathrm{Ba}$ and $\mathrm{Ca}$ columns is clearly observed, in which atomic columns of $\mathrm{CaO}$ and $\mathrm{BaO}$ appear bright and dark, respectively. Oxygen and Ti columns appear as bright. ARCC provides a direct and accurate measurement of the positions of oxygen columns that allows us to quantify $\mathrm{TiO}_{6}$ octahedral tilt angles (along the [110] direction) as a function of distance from the interface. ARCC is sensitive to the mixing between $\mathrm{Ba}$ and $\mathrm{Ca}$ at the interface, allowing us to study the relationship between the mixture and octahedral tilt angles. 


\section{References:}

[1] C. L. Jia, M. Lentzen, K. Urban, Science 299, 870-873 (2003).

[2] A. Wang, F. R. Chen, S. Van Aert, D. Van Dyck, Ultramicroscopy 110, 527-534 (2010)

[3] Research at the Electron Microscopy Center at Argonne National Laboratory is supported by the U.S. Department of Energy, Office of Science, Office of Basic Energy Sciences, under Contract No. DE-AC02-06CH11357.
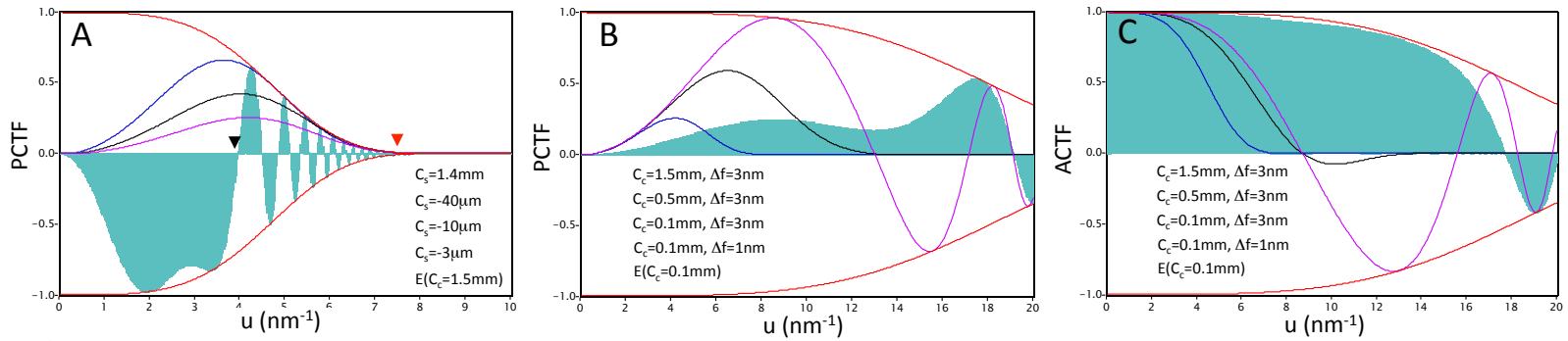

Fig. 1. (A) $C_{s}$ correction improves resolution but reduces phase contrast due to the constraint of the uncorrected $C_{c}$ damping envelope (red curve $\mathrm{C}_{\mathrm{c}}=1.5 \mathrm{~mm}$ ). For comparison, all PCTFs are plotted at Scherzer defocus for the corresponding value of $\mathrm{C}_{\mathrm{s}}$. $\mathrm{C}_{\mathrm{c}}$ correction (even just to $0.1 \mathrm{~mm}$ ) improves both $(\mathbf{B})$ PCTF and $(\mathbf{C})$ ACTF up to high spatial frequencies for small $C_{s}$ due to the extension of the $C_{c}$ damping envelope (red curve $C_{c}=0.1 \mathrm{~mm}$ ) to high spatial frequency. Adjusting defocus $\Delta \mathrm{f}$ from Scherzer defocus $3 \mathrm{~nm}$ to $1 \mathrm{~nm}$ improves the ACTF filled with green in $(\mathbf{C})$ to close to 1 over a wide range of spatial frequency up to the information limit of the microscope.

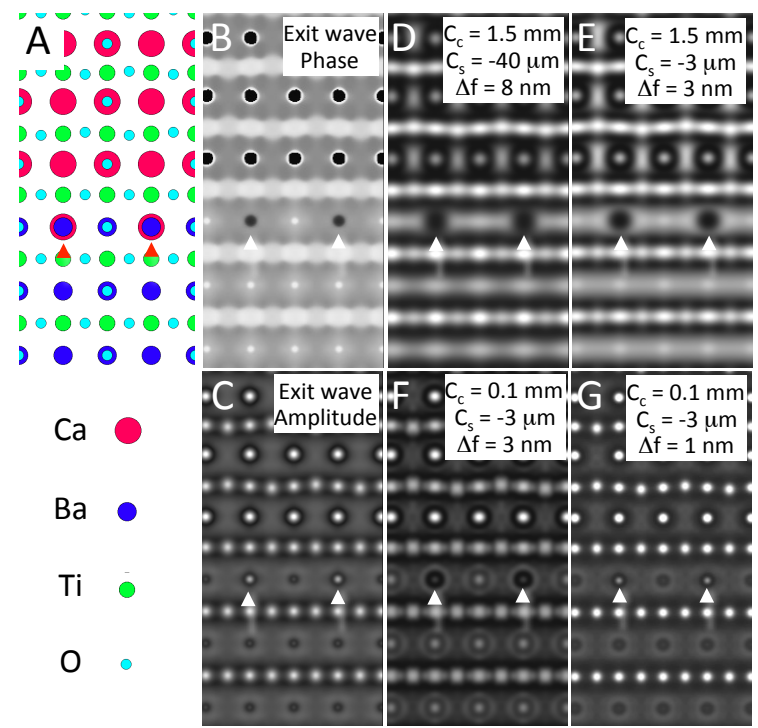

Fig. 2. Simulated images of the $\mathrm{CaTiO}_{3} / \mathrm{BaTiO}_{3}$ interface demonstrating that correction of $\mathrm{C}_{\mathrm{s}}$ and $\mathrm{C}_{\mathrm{c}}$ enables amplitude contrast HREM. (A) Atomic arrangement along [110]. (B) Phase image and (C) amplitude image of exit wave of a $9 \mathrm{~nm}$ thick sample. When $\mathrm{C}_{\mathrm{c}}$ is uncorrected $\mathrm{C}_{\mathrm{c}}=1.5 \mathrm{~mm}$ ), both simulated images using (D) $\mathrm{C}_{\mathrm{s}}=-40 \mu \mathrm{m}$ and (E) $\mathrm{C}_{\mathrm{s}}=-3 \mu \mathrm{m}$ show close correlation to phase image of the exit wave in (B). When $\mathrm{Cc}$ is corrected from $(\mathbf{E}) \mathrm{C}_{\mathrm{c}}=1.5$ $\mathrm{mm}$ to $(\mathbf{F}) \mathrm{C}_{\mathrm{c}}=0.1 \mathrm{~mm}$, simulated image in $(\mathbf{F})$ shows close correlation to the amplitude image of the exit wave in $(\mathbf{C})$. Adjusting defocus further improves the amplitude contrast in $(\mathbf{G})$.

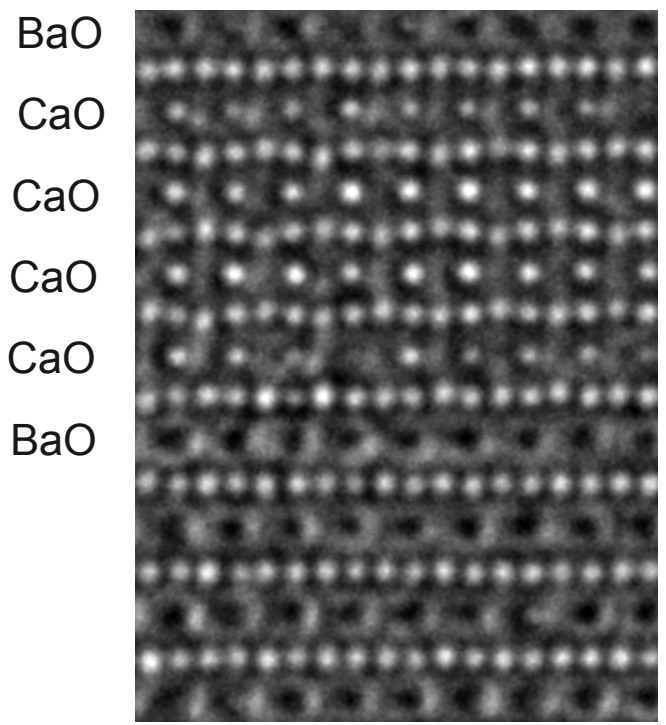

Fig. 3 Experimental image of a $\mathrm{CaTiO}_{3} / \mathrm{BaTiO}_{3}$ superlattice along [110] with an amplitude contrast HREM imaging condition of $\mathrm{C}_{\mathrm{s}}=3$ $\mu \mathrm{m}, \mathrm{C}_{\mathrm{c}}=1 \mu \mathrm{m}$. Atom columns of $\mathrm{CaO}, \mathrm{Ti}$, and $\mathrm{O}$ in this image appear as bright dots on a grey background. Atom columns of $\mathrm{BaO}$ appear darker. The image shows close correlation to the amplitude image of the exit wave. 